\title{
RELAÇÕES ENTRE PROCESSAMENTO FONOLÓGICO E LINGUAGEM ESCRITA NOS SUJEITOS COM DISTÚRBIO ESPECÍFICO DE LINGUAGEM
}

\section{Relations between phonological processing and written language in subjects with specific language impairment}

\author{
Ana Paola Nicolielo ${ }^{(1)}$, Simone Rocha de Vasconcellos Hage ${ }^{(2)}$
}

\section{RESUMO}

Objetivos: verificar a ocorrência de alterações nas habilidades da linguagem escrita e nas habilidades do PF em criança com DEL; comparar o desempenho entre crianças com DEL e com DTL quanto às diferentes habilidades do PF; verificar se há associação entre as habilidades do Processamento Fonológico e as de linguagem escrita em crianças com DEL. Método: 40 sujeitos, sendo $20 \mathrm{com}$ diagnóstico de DEL (GE) e 20 com DTL (GC) com idades entre 7 e 10 anos de ambos os sexos. Para avaliação das habilidades do PF foram aplicados os seguintes procedimentos: prova de repetição de não palavras para avaliação da Memória de Trabalho Fonológica, Teste de Nomeação Automatizada Rápida para avaliação do Acesso Lexical e Perfil de Habilidades Fonológicas para avaliação da Consciência Fonológica. A escrita e a leitura foram avaliadas por meio dos subtestes presentes no Teste de Análise de Leitura e Escrita (ditado e escrita espontânea; leitura de texto e de compreensão de texto, respectivamente). Para analise estatística foi utilizado o teste Qui Quadrado, sendo adotado nível de significância estatística $\leq 0,05$. Resultados: a existência de associação entre desempenho em provas de leitura e escrita e o desempenho em provas do PF foi confirmada. Conclusão: há associação entre desempenho em provas de leitura e escrita e provas do PF o que leva a crer que as dificuldades nas habilidades do PF podem justificar as dificuldades de linguagem escrita nesses sujeitos.

DESCRITORES: Linguagem Infantil; Transtornos do Desenvolvimento da Linguagem; Desenvolvimento da Linguagem; Criança; Avaliação de Desempenho

\section{INTRODUÇÃO}

Quando o aprendizado escolar se inicia, a criança, por sua experiência com a linguagem oral, já tem internalizada a gramática da língua, mesmo que isto seja inconsciente, pois a criança utiliza adequadamente os conhecimentos linguísticos adquiridos ao longo do aprendizado da língua materna, sem, entretanto, operar voluntariamente com eles ${ }^{1}$.

(1) Fonoaudióloga da Clínica de Fonoaudiologia da Faculdade de Odontologia de Bauru da Universidade de São Paulo FOB/USP; Mestre em Fonoaudiologia Faculdade de Odontologia de Bauru da Universidade de São Paulo - FOB/USP.

(2) Fonoaudióloga; Professora Associada do Departamento de Fonoaudiologia da Faculdade de Odontologia de Bauru da Universidade de São Paulo; Livre Docente.

Conflito de interesses: inexistente
Espera-se que, na época em que a criança inicie o aprendizado formal do código escrito, ela seja um falante bem sucedido de sua língua nativa, sendo a linguagem oral de grande importância para posterior alfabetização². Dessa forma, quando há prejuízos na linguagem oral, neste período inicial de aprendizado, a probabilidade de ocorrerem dificuldades na linguagem escrita é maior quando comparada a situações em que não há tal prejuízo ${ }^{3}$. Tal fato ocorre porque as operações de processamento de leitura e escrita são baseadas inicialmente na estrutura fonológica da linguagem oral, pelo fato da língua portuguesa ser uma língua alfabética, e envolvem a organização conceitual, a representação lexical e a memória de trabalho, que acessa e recupera as representações gráficas relacionadas aos sons da fala 4 . 
Tratando-se de prejuízos na linguagem oral, um leque de possibilidades surge. Este leque inclui desde alterações de linguagem como parte de quadros que afetam todo o desenvolvimento infantil, até alterações que acometem especificamente a linguagem nos seus diferentes níveis. Nesta última possibilidade, insere-se o quadro de Distúrbio Específico de Linguagem (DEL), cujo diagnóstico se dá mais pela exclusão de fatores do que pela inclusão ${ }^{5}$. Enquadram-se neste diagnóstico, crianças apresentam maturação de linguagem atrasada em pelo menos 12 meses em relação à idade cronológica e que não tenham déficits intelectuais ou sensoriais, distúrbios invasivos do desenvolvimento, dano cerebral evidente, além de terem condições sociais e emocionais adequadas. O quadro apresenta sintomatologia variada, podendo apresentar dificuldades somente no nível expressivo ou no nível expressivo e receptivo, com características persistentes que tendem a afetar a aprendizagem da linguagem escrita $^{6}$. Pesquisas longitudinais com crianças com DEL comprovam que a maioria destas terá dificuldades no âmbito escolar em graus variados ${ }^{7}$.

O interesse em verificar quais aspectos da linguagem oral mantém uma relação mais direta com o aprendizado da linguagem escrita é evidenciado na literatura ${ }^{8}$. O estudo dessa relação implica em admitir que essas duas modalidades de linguagem se interrelacionam ${ }^{9}$. Neste sentido, para compreensão desta relação, os estudos têm se concentrado sobre o processamento fonológico (PF), o qual se refere às operações de processamento de informação baseada na fala, ou seja, na estrutura fonológica da linguagem oral ${ }^{10}$. Nas últimas duas décadas a investigação realizada neste domínio tem acentuado a importância das habilidades que envolvem este tipo de processamento na aquisição das competências da linguagem escrita. Estas habilidades envolvem: a memória de trabalho fonológica (MTF), o acesso lexical ( $A L$ ) e a consciência fonológica (CF). Paralelamente, limitações em uma ou mais destas habilidades podem ser a justificativa dos desvios fonológicos, das dificuldades de compreensão e aquisição lexical presentes nos indivíduos com DEL, que, por conseguinte, podem justificar as alterações, muitas vezes presentes, no desenvolvimento da linguagem escrita.

Diante do contexto apresentado, este trabalho vem buscar explicações para as dificuldades de leitura e escrita no quadro de DEL, procurando verificar as relações entre essas dificuldades e o $\mathrm{PF}$, tão frequentemente encontradas nas crianças com este quadro. Dessa forma, o presente estudo tem por objetivos verificar a ocorrência de alterações nas habilidades da linguagem escrita e nas habilidades do PF em criança com DEL; comparar o desempenho entre crianças com DEL e com Desenvolvimento Típico de Linguagem (DTL) quanto às diferentes habilidades do PF; verificar se há associação entre as habilidades do PF e as de linguagem escrita em crianças com DEL.

\section{MÉTODO}

Este foi um estudo do tipo experimental, que contou com a participação de 40 sujeitos, em idade escolar, cuja faixa etária variou entre 7 e 10 anos de idade, de ambos os sexos, sendo 20 deles com diagnóstico de DEL - Grupo de Estudo (GE) - e 20 com DTL - Grupo Controle (GC), pareados de acordo com a idade cronológica e série escolar ( $1^{\text {a }}$ série - segundo ano - a $4^{a}$. série - quinto ano - do ensino fundamental). Os do GE foram selecionados dentre aqueles pacientes diagnosticados com DEL na Clínica de Fonoaudiologia da FOB/ USP. Como critério de inclusão, os sujeitos deveriam ter o diagnóstico de DEL, de acordo com os critérios propostos por Leonard (1998) ${ }^{11}$ : ter desempenho linguístico abaixo do esperado para a idade mental e cronológica, considerando-se a expressão e/ou compreensão da linguagem oral; ter limiares auditivos dentro dos padrões de normalidade; ter desempenho cognitivo de acordo com a normalidade, ou ainda, discrepância entre o desempenho das habilidades cognitivas verbais e não-verbais; ausência de problemas comportamentais e/ou emocionais; ausência de sintomatologia neurológica clássica como, por exemplo, paralisia cerebral, deficiência mental, afasia infantil (adquirida).

Tais critérios foram atendidos com base nos dados de avaliações descritos nos prontuários dos pacientes diagnosticados com DEL da Clínica de Fonoaudiologia da FOB/USP. Já os sujeitos do GC foram selecionados em duas escolas de ensino fundamental do município de Bauru, do Estado de São Paulo. Os critérios de inclusão para os sujeitos do GC foram: não apresentar queixa e/ou histórico de alterações no desenvolvimento de linguagem oral e audição, e ainda, apresentar desempenho compatível com a idade cronológica em prova de fonologia. Para atender tais critérios, primeiramente foi realizada entrevista com os professores, que foram questionados a respeito de possíveis queixas de linguagem oral e audição. Aqueles que apresentaram histórico ou queixa de dificuldades em um ou mais desses fatores foram excluídos da amostra. Os sujeitos que não tinham queixa ou histórico de alterações de comunicação oral e audição foram submetidos à Prova de Fonologia - nomeação - do Teste de linguagem infantil ABFW ${ }^{12}$.

Para se alcançar o objetivo proposto, após a verificação dos critérios de inclusão, todos os 
sujeitos da pesquisa foram submetidos a procedimentos para avaliação específica das habilidades do PF e da linguagem escrita.

Para avaliação da habilidade de MTF foi utilizada a Prova de Memória de Trabalho Fonológica $^{13}$, que tem por objetivo avaliar o número de itens que cada criança consegue reter e recuperar da memória imediata após a apresentação verbal de uma lista de não palavras e dígitos. Foi utilizado apenas o sub-teste de repetição de não palavras em que é solicitado que o sujeito repita as não palavras de forma idêntica a que lhe foi apresentada. Mediante a presença de simplificações fonológicas, no caso das crianças com DEL, estas foram previamente anotadas na ficha de resposta e não foram consideradas erros de repetição. Desta forma, anteriormente, os sujeitos com DEL, foram submetidos à prova de nomeação da Prova de Fonologia do $\mathrm{ABFW}^{12}$, para levantamento das possíveis alterações fonológicas, sendo os processos fonológicos considerados durante a análise das respostas obtidas durante a prova.

Para avaliação da habilidade de acesso lexical foi utilizado o Teste de Nomeação Automática Rápida - RAN (Rapid Automatized Naming), padronizado para a população brasileira ${ }^{14}$.

Foi utilizado o Perfil de Habilidades Fonológicas ${ }^{15}$ para avaliação da habilidade de consciência fonológica, que fornece dados sobre a capacidade do indivíduo de processar os aspectos fonológicos da língua. Ressalta-se que para os sujeitos com DEL também foram levadas em consideração as possíveis alterações fonológicas levantadas por meio da prova de nomeação da Prova de Fonologia do ABFW'11.

No que diz respeito à avaliação da habilidade de leitura e de escrita, os sujeitos foram submetidos ao Teste de Análise de Leitura e Escrita (TALE), traduzido, adaptado e padronizado para a população ${ }^{16}$. Este instrumento permite obter dados capazes de identificar características evolutivas do aprendizado da leitura e da escrita, nos diferentes níveis do ensino fundamental. Para avaliação da leitura foram utilizados apenas os sub-testes de leitura de texto e compreensão de texto, já para avaliação da escrita foram utilizados os sub-testes de ditado e escrita espontânea. Foram aplicados apenas esses subtestes visto que são os que melhor evidenciam o real desempenho tanto da leitura quanto da escrita. Ressalta-se que os textos utilizados, assim como os parâmetros de normalidade para comparação, correspondem aos quatro níveis referentes às quatro séries iniciais do Ensino Fundamental, que são: 1a série (Nível I), correspondente ao segundo ano, $2^{\mathrm{a}}$ série (Nível II), correspondente ao terceiro ano, $3^{\text {a }}$ série (Nível III), correspondente ao quarto ano e $4^{\text {a }}$ série (Nível IV), correspondente ao quinto ano do ensino fundamental.

Este trabalho foi aprovado pelo Comitê de Ética em Pesquisa da Faculdade de Odontologia de Bauru da Universidade de São Paulo (número 80/2007). Todos os pais e/ou responsáveis autorizaram a participação do (a) filho (a) na pesquisa, mediante explicação e posterior assinatura do Termo de Consentimento Livre e Esclarecido, conforme Resolução 196/96-CNS/MS.

Foram feitas análises qualitativas e estatísticas entre os grupos. Para analise estatística foi utilizado o teste Qui Quadrado. Com relação ao nível de significância estatística, para análise e discussão dos resultados foi adotado nível de significância menor ou igual a $0,05^{17}$.

\section{RESULTADOS}

Os sujeitos de cada um dos grupos foram divididos de acordo com o nível de escolaridade do ensino fundamental, sendo classificados nos seguintes níveis: nível I ( $1^{\text {a. }}$ série - segundo ano), nível II (2a. série - terceiro ano), nível III (3a․ sériequarto ano) e nível IV (4 $4^{\text {a. }}$ série - quinto ano). A quantidade de sujeitos em cada um dos níveis escolares, bem como a quantidade de sujeitos do sexo feminino e do sexo masculino, devido ao pareamento foi igual para ambos os grupos. A Figura 1 mostra o número de sujeitos em cada um dos níveis de escolaridade no GC e GE.

Para verificar a ocorrência de alterações nas habilidades da linguagem escrita e nas habilidades do PF nos sujeitos com DEL foram analisados os desempenhos em cada um dos sub-testes das provas utilizadas para avaliação das habilidades em questão. A Figura 2 demonstra a porcentagem de alteração nas provas que avaliaram as habilidades de leitura e escrita e a Figura 3 a porcentagem de alteração nas provas que avaliaram as habilidades do PF nos sujeitos com DEL.

Os sujeitos do grupo GC (DTL) não evidenciaram alteração nas habilidades de leitura e escrita, obtendo $100 \%$ de acerto nas provas que avaliaram tais habilidades. No que se refere às habilidades do PF os sujeitos do GC apresentaram $100 \%$ de acerto nas provas de consciência fonológica, nomeação rápida de dígitos, nomeação rápida de letras e nomeação rápida de cores. Nas provas de repetição de não palavras e de nomeação rápida de objetos, alguns sujeitos do GC evidenciaram desempenho aquém do esperado, como pode ser verificado na Figura 4, que também demonstra a porcentagem de ocorrência de alteração no GE (DEL), podendose comparar o desempenho dos sujeitos dos dois grupos nas provas que avaliaram o PF. 


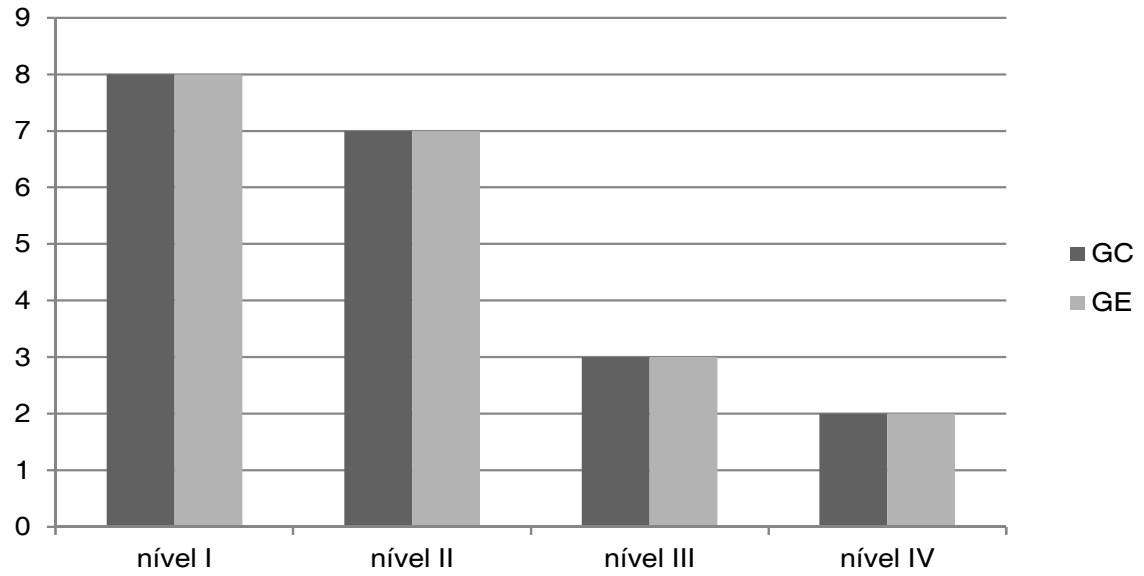

Figura 1 - Número de sujeitos do GC e do GE de acordo com os níveis de classificação de escolaridade

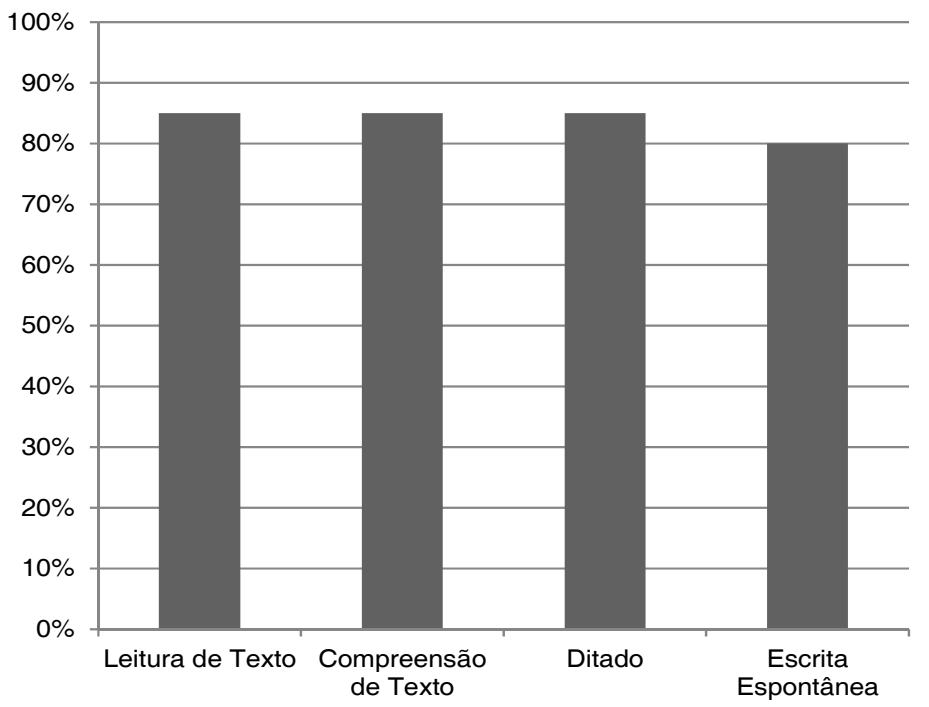

Figura 2 - Porcentagem de alteração nas habilidades de leitura e de escrita nos sujeitos com DEL

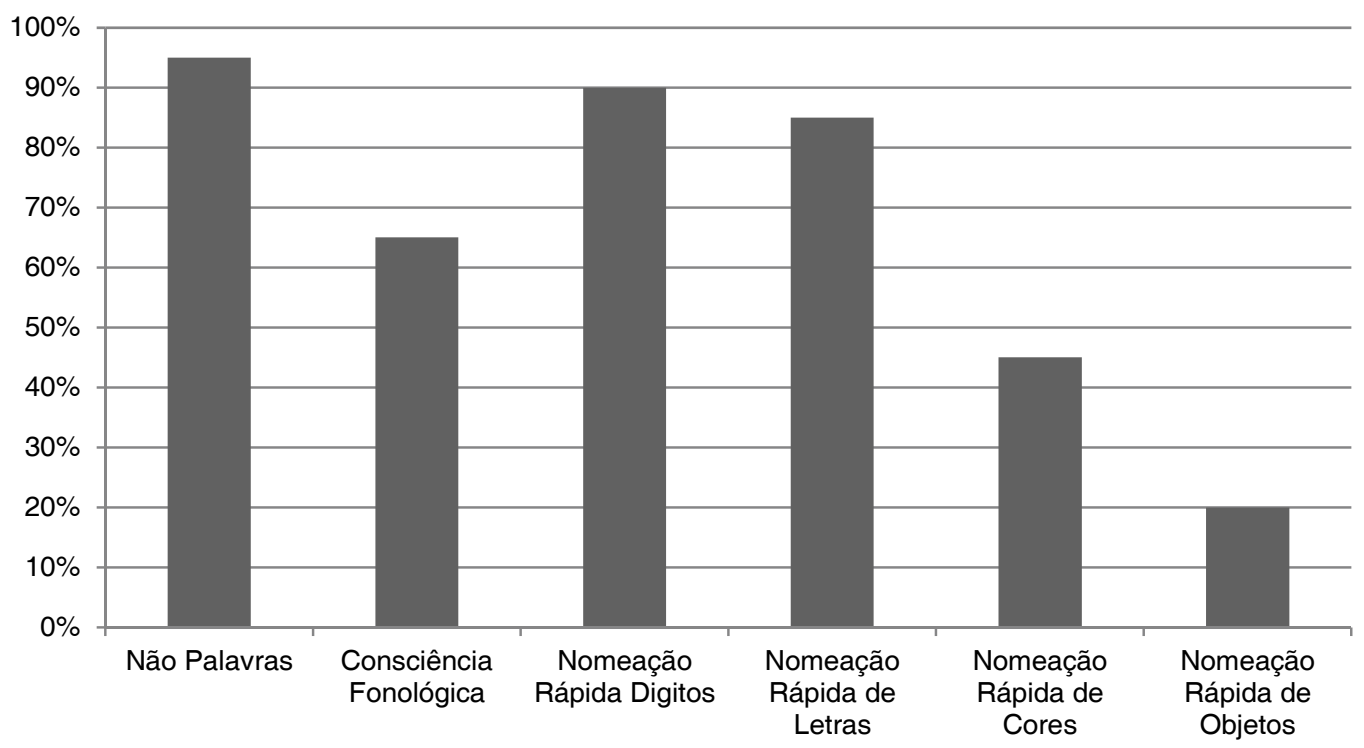

Figura 3 - Porcentagem de alteração nas habilidades do PF nos sujeitos com DEL 


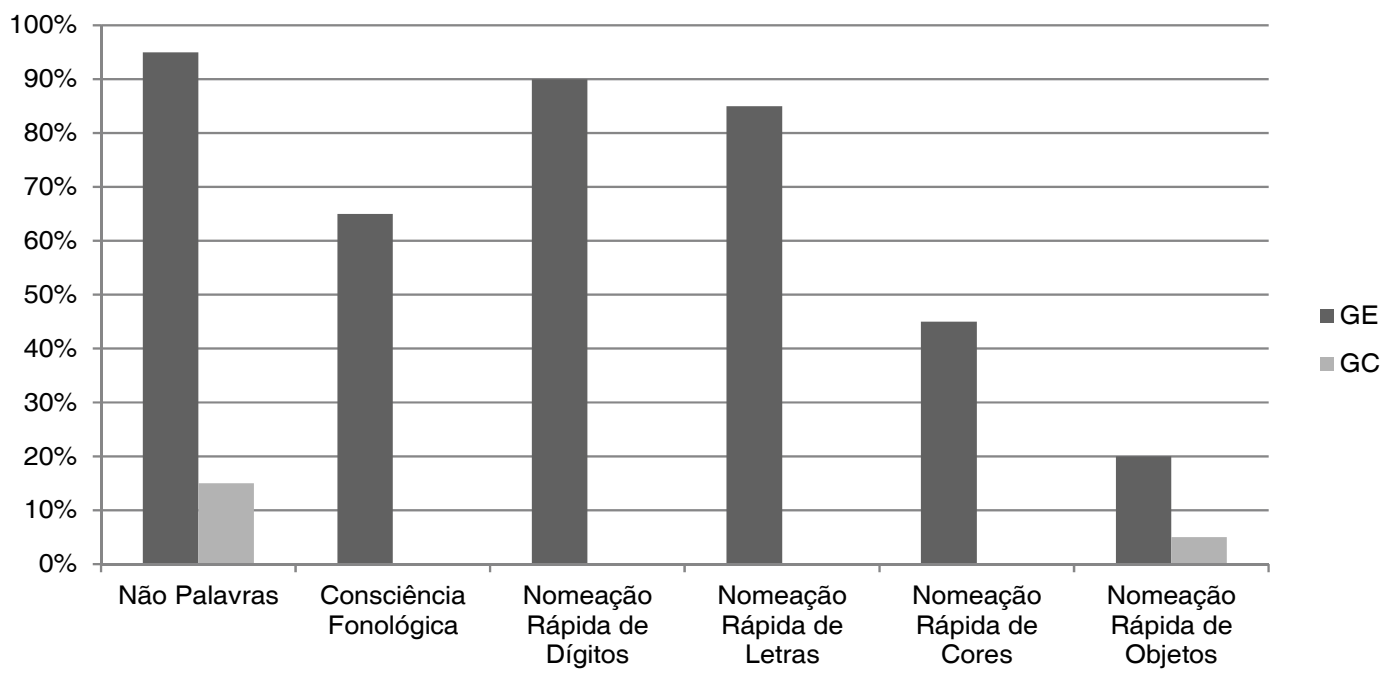

Figura 4 - Alterações no desempenho dos sujeitos do GE e do GC nas provas que avaliaram as habilidades do PF

Para estabelecer a comparação entre as crianças com DEL e com DTL quanto às habilidades do PF foram utilizados os resultados das provas aplicadas, para avaliar o PF, tanto nos sujeitos do GE (DEL) quanto nos sujeitos do GC (DTL). Foi realizada análise estatística do desempenho dos sujeitos do GE e GC em cada uma das provas do PF, por meio do teste estatístico Qui quadrado, sendo adotado nível de significância menor ou igual a 0,05.
A análise estatística demonstrou que os sujeitos do GE apresentam desempenhos significantemente piores nas habilidades do PF quando comparadas aos do GC. Apenas a prova de nomeação rápida de objetos não apresentou diferença estatística significante entre os grupos, mas o desempenho das crianças com DEL foi pior em relação às crianças com DTL, como demonstra a tabela 1.

Tabela 1 - Valores de p obtidos nas associações estatísticas entre o GE e o GC nas provas do PF

\begin{tabular}{cc}
\hline Prova & Valor de $\boldsymbol{p}$ \\
\hline Repetição de Não Palavras & 0,00000037 \\
Nomeação Rápida de Cores & 0,00044914 \\
Nomeação Rápida de Objetos & 0,07652250 \\
Nomeação Rápida de Letras & 0,00000005 \\
Nomeação Rápida de Dígitos & 0,01318437 \\
Perfil de Habilidades Fonológicas & 0,00001141 \\
\hline
\end{tabular}

Teste estatístico Qui quadrado, sendo adotado nível de significância menor ou igual a 0,05.

A fim de verificar se há associação entre as habilidades do PF e as de escrita, foram feitas associações estatísticas, por meio do teste Qui Quadrado (utilizando o mesmo nível de significância citado anteriormente), entre os desempenhos obtidos no teste TALE e os desempenhos obtidos em cada uma das provas que avaliaram as habilidades do PF nos sujeitos com DEL.

A habilidade de memória de trabalho fonológica, avaliada por meio da prova de repetição de não palavras apresentou associação estatisticamente significante com os sub-testes de leitura e de escrita, assim como a habilidade de consciência fonológica, avaliada por meio do Perfil de Habilidades Fonológicas (tabela 2 e 3). Já para a habilidade de acesso lexical houve associação estatística entre as provas de nomeação rápida de dígitos e letras com as provas de leitura do TALE (tabela 2) e entre as provas de nomeação rápida de dígitos e letras com a prova de ditado do TALE (tabela 3). 
Tabela 2 - Associações estatisticamente significantes entre o desempenho nas provas que avaliam as habilidades do processamento fonológico com o desempenho nos sub-testes de leitura do TALE dos sujeitos do GE (DEL)

\begin{tabular}{ccc}
\hline Provas & sub-testes Leitura & Valor de $\boldsymbol{p}$ \\
\hline Repetição de Não Palavras & Leitura de Texto & 0,01207380 \\
Repetição de Não palavras & Compreensão de Texto & 0,01207380 \\
Perfil de Habilidades Fonológicas & Leitura de Texto & 0,01046121 \\
Perfil de Habilidades Fonológicas & Compreensão de Texto & 0,01046121 \\
Nomeação Rápida de Dígitos & Leitura de Texto & 0,04685418 \\
Nomeação Rápida de Dígitos & Compreensão de Texto & 0,04685418 \\
Nomeação Rápida de Letras & Leitura de Texto & 0,00656070 \\
Nomeação Rápida de Letras & Compreensão de Texto & 0,00656070 \\
\hline
\end{tabular}

Teste estatístico Qui quadrado, sendo adotado nível de significância menor ou igual a 0,05.

Tabela 3 - Associações estatisticamente significantes entre o desempenho nas provas que avaliam as habilidades do processamento fonológico com o desempenho nos sub-testes de escrita do TALE dos sujeitos do GE (DEL)

\begin{tabular}{ccc}
\hline Provas & Sub-testes Escrita & Valor de $\boldsymbol{p}$ \\
\hline Repetição de Não Palavras & Escrita Espontânea & 0,00208440 \\
Repetição de Não Palavras & Ditado & 0,01459333 \\
Perfil de Habilidades Fonológicas & Escrita Espontânea & 0,04220450 \\
Perfil de Habilidades Fonológicas & Ditado & 0,010461214 \\
Nomeação Rápida de Dígitos & Ditado & 0,04685418 \\
Nomeação Rápida de Letras & Ditado & 0,00656070 \\
\hline
\end{tabular}

Teste estatístico Qui quadrado, sendo adotado nível de significância menor ou igual a 0,05.

\section{DISCUSSÃO}

A aprendizagem do sistema alfabético como é o caso da Língua Portuguesa, em que se estabelece a relação entre o grafema e o fonema, depende da capacidade do indivíduo em processar a fala. Não é de hoje que estudos vêm sendo desenvolvidos na tentativa de compreender a relação existente entre a linguagem oral e a linguagem escrita ${ }^{18,19}$. A compreensão desta relação tem sido mais explícita no âmbito das alterações, como é o caso do DEL.

A maioria dos sujeitos com DEL apresentou desempenho deficitário nas provas que avaliaram a linguagem escrita (gráfico 2). Nas provas de leitura, compreensão de texto e ditado, $85 \%$ desses sujeitos (17) apresentaram baixo desempenho, e $80 \%$ (16) apresentou desempenho deficitário na escrita espontânea. Analisando-se individualmente as crianças, somente uma apresentou desempenho adequado em todas as provas, estando ela na segunda série. Assim, a ocorrência de alterações na linguagem escrita na grande maioria dos sujeitos com DEL na amostra estudada foi constatada. Estima-se o DEL afete entre $5-7 \%$ das crianças em idade escolar ${ }^{11}$.

A ocorrência de alterações de linguagem escrita nos sujeitos com DEL tem sido descrita na literatura ${ }^{20-22}$. Uma das justificativas para as dificuldades apresentadas na linguagem escrita por estes sujeitos é a influência que o aspecto fonológico da linguagem oral exerce no desenvolvimento da linguagem escrita, principalmente devido ao fato de a Língua Portuguesa ser uma língua alfabética, em que um grafema é relacionado a um fonema. Estudos apontam alta prevalência de ocorrência de alteração fonológica em crianças com 7 anos de idade, fase em que se inicia o aprendizado da leitura e da escrita ${ }^{23}$, assim como outros que apontam a influência das alterações fonológicas nas ocorrências de alterações do desenvolvimento da linguagem escrita ${ }^{24}$. Sendo assim, alterações na linguagem escrita são de certa forma, esperadas nos sujeitos com DEL, devido à presença, nesses quadros, de alterações persistentes na linguagem 
oral, principalmente relacionada ao aspecto fonológico, em que podem ocorrer processos fonológicos do desenvolvimento da linguagem e de processos fonológicos idiossincráticos concomitantemente ${ }^{25}$.

A partir dos anos 70, a literatura começou a evidenciar estudos que revelaram a importância do PF para aquisição da linguagem escrita ${ }^{26}$, sendo assim a teoria da Hipótese do Déficit Visual27, relacionada ao processamento visual, foi substituída pela teoria da Hipótese do Déficit Fonológico para explicar a natureza subjacente dos problemas de leitura e de escrita.

A presença de alteração nas habilidades do PF foi constatada na grande maioria dos sujeitos com DEL no presente estudo (gráfico 3), assim como constatado em outros trabalhos presentes na literatura, que destacam o papel crucial deste tipo de processamento no desenvolvimento da linguagem escrita ${ }^{28-30}$. Ainda no presente estudo, ficou evidente que os sujeitos com DEL apresentaram, em maior ou menor grau, alteração nas três habilidades do PF. Na literatura os estudos, em sua maioria, realizam investigações das habilidades que compõem o PF separadamente, sendo dificilmente encontrados trabalhos que estudam as três habilidades conjuntamente. Sendo assim, trabalhos pioneiros em estudar a MTF, em sujeitos com DEL ${ }^{31}$, bem como aqueles que apontam, igualmente, piores desempenhos de crianças com prejuízos na linguagem em tarefas de repetição de não palavras, evidenciam a importância clínica destes tipos de tarefas em casos de distúrbio de linguagem. Tal fato evidencia que o desempenho em tais tarefas pode ser um marcador lingüístico nesses quadros e, além de fornecer valiosas informações a respeito das capacidades lingüísticas dos sujeitos com e sem distúrbio de linguagem, pode predizer possíveis déficits na linguagem oral e linguagem escrita desses sujeitos ${ }^{32}$.

Os estudos que investigam a habilidade de CF também corroboram com os achados do presente estudo, apontando alterações em tal habilidade nos sujeitos com DEL Alterações na aquisição e desenvolvimento da linguagem prejudicam, consequentemente, o desenvolvimento da habilidade de CF e, por conseguinte, prejudicam o desenvolvimento da linguagem escrita ${ }^{33}$. Outra habilidade do PF também analisada neste estudo, o $\mathrm{AL}$, um processo que permite que o indivíduo acesse as informações fonológicas estocadas no léxico mental, por meio dos processos da memória de longo prazo, com enorme facilidade e rapidez. Entretanto, no presente estudo, tal facilidade e rapidez não foram evidenciadas nos desempenhos dos sujeitos com DEL em testes de nomeação rápida (Figura 3). Outros estudos também evidenciam que a velocidade de processamento nos sujeitos com DEL é, geralmente, mais lenta ${ }^{34}$, havendo, também, estudos que relacionam o baixo desempenho em tarefas de nomeação automatizada rápida, sendo tal desempenho característico de grupos de indivíduos com dificuldades de leitura ${ }^{35}$

Diante do exposto, fica evidente na literatura, em trabalhos realizados nas últimas três décadas, o papel crucial desempenhado pelo PF na aquisição e desenvolvimento da linguagem escrita, corroborando com o achado do presente estudo que confirmou a existência de associação entre desempenho em provas de leitura e escrita e desempenho em provas do PF. Tal achado fornece indícios para a premissa de que a presença de alterações na linguagem escrita está atrelada às dificuldades presentes nas habilidades do PF. Tal associação sugere a relação entre PF, linguagem oral e linguagem escrita, sendo que alteração neste processamento pode ser a origem das dificuldades lingüísticas e de aprendizagem das crianças com DEL. Dessa forma, quando há alteração na aquisição e desenvolvimento da linguagem oral, principalmente no que se refere ao aspecto fonológico, provavelmente também estará presente alterações nas habilidades que compõem o PF o que, conseqüentemente, acarretará em prejuízos na aquisição e desenvolvimento da linguagem escrita.

\section{CONCLUSÃO}

A ocorrência de alterações nas habilidades da linguagem escrita e nas habilidades do PF nos sujeitos com DEL foi confirmada, sendo o desempenho dos sujeitos com DEL significantemente pior em relação aqueles com Desenvolvimento Típico de Linguagem. Foi confirmada a associação entre desempenho em provas de leitura e escrita e provas do PF. As dificuldades nas habilidades do PF podem justificar as dificuldades de linguagem escrita presentes nesses sujeitos. A associação entre habilidades do PF e leitura e escrita ocorre pelo fato de que, assim como ocorre no processamento da linguagem oral, para que o indivíduo expresse a linguagem em sua forma escrita também utilizará as habilidades que compõem o PF. Deverá acessar as informações, na memória de longo prazo, por meio do acesso lexical, que foram estocadas na memória de longo prazo por meio de todo o processo que envolve a Memória de trabalho fonológica, além de também fazer uso da consciência fonológica. Se tais habilidades estão comprometidas, consequentemente a linguagem escrita também está sujeita a apresentar alterações. 


\section{ABSTRACT}

Purpose: to check the occurrence of alterations in language skills and writing skills of Phonological Processing (PP) in children with SLI, compare the performance between children with SLI and Typical Language Development on the different abilities of PP; check for association between PP and skills of written language in children with SLI. Method: 40 subjects, 20 with SLI (EG) and 20 with TLD (CG) aged between 7 and 10 year old for both genders. To assess the abilities of PP we applied the following tests: proof of non word repetitions to assess phonological working memory (PWM), Rapid Automated Naming test (RAN) for evaluating Lexical Access (LA) and Phonological Abilities Profile for assessing phonological awareness (PA). Writing and reading were evaluated using the subtests present in Test for Análising Reading and Writing Skills (dictation and spontaneous writing, reading text and reading comprehension, respectively). For statistical analysis we used the Chi-Square test, with significance level $\leq 0.05$. Results: an association between performance on tests of reading and writing and performance on tests of PP was confirmed. Conclusion: there is an association between performance in reading and writing tests of PP which suggests that the difficulties in the abilities referring to PF may explain the difficulties of written language regarding these subjects.

KEYWORDS: Child Language; Language Development Disorders; Language Development; Child; Employee Performance Appraisal

\section{REFERÊNCIAS}

1. Serafim MS de. Práticas de escrita na sala de aula: a escrita como possibilidade de interação. Rev. De Divulgação Científica em Língua Portuguesa, Linguistica e Literatura [periódico da internet]. 2010. [acesso em 2011, 06 de abril]; 13: [aprox. 19 p]. Disponível em: http://letramagna.com/Artigo09_13. pdf

2. Capovilla AGS; Dias NMD. Habilidades de linguagem oral e sua contribuição para a posterior aquisição de leitura. PSIC Rev. de Psicologia. 2008;9(2):135-44.

3. American Speech-Language-Hearing Association (ASHA). Speech-Language Pathologists' Role in Reading and Writing. [2008]. [citado 2010 Abr 06]. Disponível em: http://www.asha.org/about/news/ tipsheets/SLPs-Role.htm.

4. Mezzomo CL; Mota HB; Dias RF.Desvio Fonológico: aspectos sobre produção, percepção e escrita. Rev Soc Bras Fonoaudiol. 2010;15(4):554-60.

5. Rocha LC; Befi-Lopes D M; Rodrigues A. Análise pragmática das respostas de crianças com e sem distúrbio específico de linguagem. Rev. Pró-Fono. 2006;18(3):229-38.

6. Hage SRV; Cendes F; Montenegro MA; Abramides DV; Guimarães CA; Guerreiro MM. Specific language impairment: linguistic and neurobiological aspects. Arq. Neuro-Psiquiatr. [serial on the Internet]. 2006 June [cited $2011 \mathrm{Apr}$ 07]; 64(2a): 173-80. Available from: http://www.
scielo.br/scielo.php?script=sci_arttext\&pid=S0004282X2006000200001\&lng=en. doi: 10.1590/ S0004-282X2006000200001.

7. Conti-Ramsden G, Botting N, Simkin Z, Knox $E$. Follow-up of children attending infant language units: outcomes at 11 years of age. Int $\mathrm{J}$ Lang Commun Disord. 2001; 36(2):207-19.

8. Capellini SA; Conrado TLBC. Desempenho de Escolares com e sem dificuldades de aprendizagem de ensino particular em habilidade fonológica, nomeação rápida, leitura e escrita. Rev. CEFAC. 2009;11(2):183-93.

9. Gindri G; Keske-Soares M; Mota HB. Memória de trabalho, consciência fonológica e hipóteses de escrita. Pró fono Rev. de Atualização Científica. 2007;19(3):313-22.

10. Soares HMA, Reis MPR, Aquino KO, Assis JR. Diagnóstico precoce da dislexia: importância da equipe multidisciplinar. R. Min. Edc. Fís. 2010;5:209-18.

11. Leonard LB. Children with Specific Language Impairment. 1ed. Boston: MIT Press, 1998.

12. Wertzner HF. Fonologia. In: Andrade CRF, BefiLopes DM, Fernandes FDM, Wertzner HF. ABFW: Teste de linguagem infantil nas áreas de Fonologia, Vocabulário, Fluência e Pragmática. 2ed. São Paulo: Pró-Fono, 2000. p. 5-31.

13. Hage SRV, Grivol MA. Desempenho de crianças normais falantes do português em prova de memória de trabalho fonológica. Cad.Comun. Ling. 2009; 1:11-22.

14. Ferreira TL, Capellini SA, Ciasca SM, Tonelotto JMF. Desempenho de escolares leitores proficientes 
no teste de nomeação automatizada rápida RAN. Temas Desenvolv. 2003; 12(69):26-32.

15. Alvarez AMMA, Carvalho IAM, Caetano AL. Perfil de Habilidades Fonológicas. 2ed. São Paulo: Via Lettera; 2004.

16. Anderle STS. Teste de Análise de Leitura e Escrita Tradução, Adaptação e Validação [dissertação]. Florianópolis (SC): Universidade do Sul de Santa Catarina; 2005.

17. Rosner B. Fundamentals of Biostatistics. 5th ed. Duxbury: Pacific Grove. 2000.

18. Miilher LP, Ávila CRB. Variáveis linguísticas e de narrativas no distúrbio de linguagem oral e escrita. Pro fono. 2006;18(2) 177-88.

19. Zorzi JL, Ciasca SM. Análise dos erros ortográficos em diferentes problemas de aprendizagem. Rev. Cefac. 2009;11(3):406-16.

20. Evans JL; Saffran JR. Robe-Torres K. Statistical learning in children with Specific Languaje Impairment. J Speech Lang Hear Res. 2009;52:321-35.

21. Nicolielo AP, Fernandes GB, Garcia VL, Hage SRV. Desempenho escolar de crianças com distúrbio específico de linguagem: relações com habilidades metafonológicas e memória de curto prazo. Rev. soc. bras. Fonoaudiol. 2008;13:246-50. 22. Dockrell JE, Lindsay G, Connelly V. The impact of specific language impairment on adolescents' written text. Exceptional children. 2009; 15(4):426-7. 23. Patah LK; Takiuchi. Prevalência das alterações fonológicas e uso dos processos fonológicos em escolares aos 7 anos. Rev. Cefac. 2008;10(2):158-67.

24. Goulart BNG de; Chiari BM. Prevalência de desordens de fala em escolares e fatores associados. Rev. Saúde Pública [periódico na Internet]. 2007 Out [citado 2011 Abr 07] ; 41(5): 726-31. Disponível em: http://www.scielo.br/scielo.php?script=sci arttext\&pid=S0034-89102007000500006\&lng=pt. doi: 10.1590/S0034-89102007000500006.

http://dx.doi.org/10.1590/S1516-18462011005000086

RECEBIDO EM: 02/03/2011

ACEITO EM: 04/07/2011

Endereço para correspondência:

Ana Paola Nicolielo

Clínica de Fonoaudiologia

Al. Octávio Pinheiro Brisola, 9-75

Bauru - SP - Brasil

CEP:17012-901

E-mail: anapaolanicolielo@yahoo.com.br
25. Rondon S, Befi-Lopes DM. Características iniciais da comunicação verbal de pré-escolares com Alterações Específicas do Desenvolvimento da Linguagem em fala espontânea. Rev. Soc. Bras. Fonoaudiol; 2010; 15(3): 415-20.

26. Liberman I, Shankweiler D, Fischer F, carter B. Explicit syllable and phoneme segmentation in the young child. Journal of experimental child psychology. 1974;18:201-12.

27. Ajuriaguerra $\mathrm{J}$ de. Les dyslexies d'évolution, problème théoriques et pratiques de neuropsychiatrie infantile. Annales médico-psychologiques. 1953; 1 : 540-51.

28. Navas ALGP, Pinto JCBR, Dellisa PRR. Avanços no conhecimento do processamento da fluência em leitura: da palavra ao texto. Rev Soc Bras Fonoaudiol. 2009; 14(3): 553-9.

29. Affonso MJCO, Piza CMJT, Barbosa ACC, Macedo EC. Avaliação de escrita na dislexia do desenvolvimento: tipos de erros ortográficos em prova de nomeação de figuras por escrita. Rev. CEFAC [periódico na internet]. 2010 Out. [acesso em 2011, fev 03]; [aproximadamento 8]. ISSN 15161846. doi: 10.1590/S1516-18462010005000117. http://www.scielo.br/pdf/rcefac/2010nahead/54-10. pdf

30. Gindri G.; Keske-Soares M.; MOTA, H. B. Working memory, phonological awareness and spelling hypothesis (original title: Memória de trabalho, consciência fonológica e hipótese de escrita). Rev. Pró-Fono. 2007;19(3)3:313-22.

31. Gathercole S, Baddley AD. Phonological memory deficits in language disordered children: is there a causal connection? J Mem Lang.1990; 29(3): 336-60.

32. Lobo FS; Acrani IO; Ávila CRB de. Tipo de estímulo e memória de trabalho fonológica. Rev. Cefac. 2008; 10(4):461-70.

33. Nicolielo AP. Relações entre processamento fonológico e alterações de leitura e escrita em crianças com Distúrbio Específico de Linguagem [dissertação]. Bauru-SP: Universidade de São Paulo. Faculdade de Odontologia de Bauru. Departamento de Fonoaudiologia. 2009.

34. Nicolielo AP, Hage SRV. Desempenho em Prova de Recodificação Fonológica de Acesso ao Léxico em Crianças com Distúrbio Específico de Linguagem com Dificuldades de Aprendizagem. In: 16 Congresso Brasileiro de Fonoaudiologia; 2008 set. 24-27; Campos do Jordão, SP. Anais - Rev. Soc. Bras. Fonoaud. - suplemento Especial. São Paulo: SBFa, 2008.

35. Capellini SA; Lanza SC. Desempenho de Escolares em consciência fonológica, nomeação rápida, leitura e escrita. Pró fono Rev. de Atualização Científica. 2010;22(3):293-344. 